\title{
Modified Sampling Strategies Using Correlation Coefficient for Estimating Population Mean
}

\author{
Shashi Bhushan* and Arvind Pandey \\ Mizoram University
}

\begin{abstract}
This paper proposes two sampling strategies based on the modified ratio estimator using the population mean of auxiliary variable and population correlation coefficient between the study variable and the auxiliary variable by Singh and Tailor (2003) for estimating the population mean (total) of the study variable in a finite population. A comparative study is made with usual sampling strategies and some concluding remarks are given. Finally, an empirical study is included as an illustration which shows that the proposed sampling strategies are better than Singh and Tailor estimator both in terms of unbiasedness and lesser mean square error.
\end{abstract}

Keywords. Ratio estimator; unbiasedness; mean square error; range prior information.

MSC 2010: 62D05.

\section{Introduction}

The use of population correlation coefficient in deriving the efficiency condition of the ratio estimator and product estimator has been discussed by various authors including, Cochran (1977), Sukhatme and Sukhatme (1997) and Singh and Chaudhary (2002). Recently, Singh and Tailor (2003) used the known population correlation coefficient to increase the efficiency of the estimation procedure. Let $\rho$ be the population correlation coefficient between

* Corresponding author 
the study variable $y$ and the auxiliary variable $x$. Assuming $\rho$ and population mean $\bar{X}$ of $x$ to be known, Singh and Tailor (2003) proposed modified ratio estimator and modified product estimator for estimating the population mean $\bar{Y}$ of $y$ given by $\bar{y}_{S R}=\bar{y}(\bar{X}+\rho) /(\bar{x}+\rho)$ and $\bar{y}_{S P}=\bar{y}(\bar{x}+\rho) /(\bar{X}+\rho)$ respectively; where $\bar{y}$ and $\bar{x}$ be the sample means of the $y$ and $x$ respectively. Singh and Tailor (2003) estimators were found efficient but biased. The expressions of bias and mean square error of $\bar{y}_{S R}$ and $\bar{y}_{S P}$ under Simple Random Sampling without Replacement (SRSWOR) are given by

$$
\begin{aligned}
\operatorname{Bias}\left(\bar{y}_{S R}\right) & =\gamma_{n} \bar{Y}\left(\nu^{2} C_{x}^{2}-\rho \nu C_{x} C_{y}\right) \\
\operatorname{MSE}\left(\bar{y}_{S R}\right) & =\gamma_{n} \bar{Y}^{2}\left\{C_{y}^{2}+\nu C_{x}^{2}(\nu-2 K)\right\}
\end{aligned}
$$

and

$$
\begin{aligned}
\operatorname{Bias}\left(\bar{y}_{S P}\right) & =\gamma_{n} \bar{Y} \rho \nu C_{x} C_{y} \\
\operatorname{MSE}\left(\bar{y}_{S P}\right) & =\gamma_{n} \bar{Y}^{2}\left\{C_{y}^{2}+\nu C_{x}^{2}(\nu+2 K)\right\}
\end{aligned}
$$

respectively; where $\gamma_{n}=\left(1-f_{n}\right) / n, f_{n}=n / N, C_{x}$ and $C_{y}$ are the population coefficient of variation of $x$ and $y$, respectively, $\nu=\bar{X} /(\bar{X}+\rho)$ and $K=$ $\rho C_{y} / C_{x}$.

In this paper, we propose some modified sampling strategies such that the estimator of population mean $\bar{Y}$ is

$$
\bar{y}_{S A}=\frac{\bar{y}(\bar{X}+\rho)}{A(\bar{x}+\rho)+(1-A)(\bar{X}+\rho)}
$$

where $A$ is a characterizing scalar to be chosen suitably.

This estimator can be seen as the combination of the Walsh (1970) estimator and Singh and Tailor (2003). Note that the proposed estimator reduces to $\bar{y}_{S R}$ if $A=1$. We now consider this estimator under the following sampling schemes:

1. Simple random sampling without replacement along with the JackKnife technique and denote the resulting estimator as $\bar{y}_{S J}$.

2. Midzuno (1952) - Lahiri (1951) - Sen (1952) type sampling scheme and denote the resulting estimator by $\bar{y}_{S M}$.

Both the sampling strategies aim at getting some classes of better sampling strategies than the existing ones in the sense of unbiasedness and lesser mean square error. 
Consider estimator $\bar{y}_{S A}$ under SRSWOR and denote it by $\bar{y}_{S S}$. Let $\bar{y}=$ $\bar{Y}+e_{0}$ and $\bar{x}=\bar{X}+e_{1}$ such that

$$
E_{S}\left(e_{0}\right)=E_{S}\left(e_{1}\right)=0
$$

where $E_{S}$ denotes the expectation under SRSWOR.

Putting these values in the estimator and simplifying, we have

$$
\bar{y}_{S S}-\bar{Y}=\frac{e_{0}-A \bar{Y} e_{1}}{(\bar{X}+\rho)}+\frac{A^{2} \bar{Y}_{e_{1}}^{2}}{(\bar{X}+\rho)^{2}}-\frac{A e_{0} e_{1}}{(\bar{X}+\rho)}+\ldots
$$

Taking expectation on both sides and using (4) we get

$$
\operatorname{Bias}\left(\bar{y}_{S S}\right)=E_{s}\left(\bar{y}_{S S}\right)-\bar{Y}=\bar{Y}\left\{\frac{A^{2} E_{s}\left(e_{1}^{2}\right)}{(\bar{X}+\rho)^{2}}-\frac{A E_{s}\left(e_{0} e_{1}\right)}{(\bar{X}+\rho)}\right\}
$$

(up to the first order of approximation). Since

$$
E_{s}\left(e_{0}^{2}\right)=\gamma_{n} \bar{Y}^{2} C_{y}^{2}, \quad E_{s}\left(e_{1}^{2}\right)=\gamma_{n} \bar{X}^{2} C_{x}^{2}, \quad E_{s}\left(e_{0} e_{1}\right)=\gamma_{n} \bar{X} \bar{Y} \rho C_{x} C_{y}
$$

Therefore,

$$
\operatorname{Bias}\left(\bar{y}_{S S}\right)=\gamma_{n} \bar{Y}\left(A^{2} \nu^{2} C_{x}^{2}-A \nu \rho C_{x} C_{y}\right)=\gamma_{n} \bar{Y}\left\{A \nu C_{x}^{2}(A \nu-K)\right\}
$$

Now for mean square error consider (7) up to the first order of approximation

$$
\begin{aligned}
\operatorname{MSE}\left(\bar{y}_{S S}\right) & =E_{s}\left(\bar{y}_{S S}-\bar{Y}\right)^{2} \\
& =E_{s}\left\{e_{0}-\frac{A \bar{Y} e_{1}}{(\bar{X}+\rho)}\right\}^{2} \\
& =E_{s}\left(e_{0}^{2}\right)+\frac{A^{2} \bar{Y}^{2} E_{s}\left(e_{1}^{2}\right)}{(\bar{X}+\rho)^{2}}-\frac{2 A \bar{Y} E_{s}\left(e_{0} e_{1}\right)}{(\bar{X}+\rho)} \\
& =\gamma_{n} \bar{Y}^{2}\left(C_{y}^{2}+A^{2} \nu^{2} C_{x}^{2}-2 A \nu \rho C_{x} C_{y}\right) \\
& =\gamma_{n} \bar{Y}^{2}\left\{C_{y}^{2}+\nu C_{x}^{2}\left(A^{2} \nu-2 A K\right)\right\}
\end{aligned}
$$

It is interesting to note that on putting $A=1$ in (7) and (8) we get expressions of bias and mean square error of $\bar{y}_{S R}$; the optimizing value of the characterizing scalar $A$ is given by $A=K / \nu=A_{\text {opt }}$ (say) and the minimum mean square error under optimizing value of $A=A_{\text {opt }}$ is

$$
\operatorname{MSE}\left(\bar{y}_{S S}\right)=\gamma_{n} \bar{Y}^{2}\left(1-\rho^{2}\right) C_{y}^{2}
$$

which is same as the mean square error of the linear regression estimator. Also note that $\operatorname{Bias}\left(\bar{y}_{S S}\right)=0$ under the optimizing value of $A$. 


\section{The Jackknife Estimator}

Let us now apply Quenouille's (1956) method of Jackknife such that the sample of size $n=2 m$ from a population of size $N$ is split up at random into two sub samples of size $m$ each. For further details one may refer to Gray and Schucany (1972). Let us define

$$
\begin{aligned}
\bar{y}_{S S}^{(i)} & =\frac{\bar{y}_{i}(\bar{X}+\rho)}{A\left(\bar{x}_{i}+\rho\right)+(1-A)(\bar{X}+\rho)}, \quad i=1,2, \\
\bar{y}_{S S}^{(3)} & =\frac{\bar{y}(\bar{X}+\rho)}{A(\bar{x}+\rho)+(1-A)(\bar{X}+\rho)}
\end{aligned}
$$

where $A$ is the characterizing scalar to be chosen suitably such that $S=$ $S_{1}+S_{2}, S_{1}$ and $S_{2}$ be the two sub samples of size $m$ each and + denotes the disjoint union. $\bar{y}_{1}, \bar{y}_{2}$ and $\bar{y}$ denote the sample means based on two sub samples of size $m$ and the entire sample of size $n=2 m$ for characteristic $y$. $\bar{x}_{1}, \bar{x}_{2}$ and $\bar{x}$ denote the sample means based on two sub samples of size $m$ and the entire sample of size $n=2 m$ for characteristic $x$.

It can be easily seen that

$$
\begin{aligned}
& \operatorname{Bias}\left(\bar{y}_{S S}^{(i)}\right)=\gamma_{m} \bar{Y}\left\{A^{2} \nu^{2} C_{x}^{2}-A \nu \rho C_{x} C_{y}\right\}, \quad i=1,2, \\
& \operatorname{Bias}\left(\bar{y}_{S S}^{(3)}\right)=\gamma_{2 m} \bar{Y}\left\{A^{2} \nu^{2} C_{x}^{2}-A \nu \rho C_{x} C_{y}\right\}=B_{1} \text { (say) }
\end{aligned}
$$

Let us define ${\widehat{\overline{Y_{S S}}}}^{\prime}=\left(\bar{y}_{S S}^{(1)}+\bar{y}_{S S}^{(2)}\right) / 2$ as an alternative estimator of the population mean $\bar{Y}$.

The bias of $\widehat{\bar{Y}}_{S S}^{\prime}$ is

$$
\operatorname{Bias}\left(\widehat{\bar{Y}}_{S S}^{\prime}\right)=\gamma_{m} \bar{Y}\left\{A^{2} \nu^{2} C_{x}^{2}-A \nu \rho C_{x} C_{y}\right\}=B_{2} \text { (say) }
$$

We propose the Jackknife estimator $\bar{y}_{S J}$ for estimating population mean $\bar{Y}$ given by

$$
\bar{y}_{S J}=\frac{\bar{y}_{S S}^{(3)}-R{\widehat{\overline{Y_{S S}}}}^{\prime}}{1-R}
$$

where $R=B_{1} / B_{2}$.

Taking expectation of (13) and using (11) and (12) we obtain $E\left(\bar{y}_{S J}\right)=$ $\bar{Y}$ showing that the Jackknife estimator $\bar{y}_{S J}$ is an unbiased estimator of 
population mean $\bar{Y}$ to the first order of approximation.

Consider

$$
\begin{aligned}
\mathrm{MSE}= & E\left\{\frac{\left(\bar{y}_{S S}^{(3)}-R{\widehat{\overline{Y_{S S}}}}^{\prime}\right)}{(1-R)-\bar{Y}}\right\}^{2} \\
= & (1-R)^{-2}\left\{E\left(\bar{y}_{S S}^{(3)}-\bar{Y}\right)^{2}+R^{2} E\left({\widehat{\bar{Y}_{S S}}}^{\prime}-\bar{Y}\right)^{2}\right. \\
& \left.-2 R E\left(\bar{y}_{s S}^{(3)}-\bar{Y}\right)\left(\widehat{\bar{Y}}_{S S}^{\prime}-\bar{Y}\right)\right\}
\end{aligned}
$$

Also,

$$
E\left(\bar{y}_{S S}^{(3)}-\bar{Y}\right)^{2}=\operatorname{MSE}\left(\bar{y}_{S S}^{(3)}\right)=\gamma_{2 m} \bar{Y}^{2}\left\{C_{y}^{2}+\nu C_{x}^{2}\left(A^{2} \nu-2 A K\right)\right\}
$$

Further,

$$
\begin{aligned}
E\left(\widehat{\bar{Y}}_{S S}^{\prime}-\bar{Y}\right)^{2}= & E\left\{\frac{\left(\bar{y}_{S S}^{(1)}+\bar{y}_{S S}^{(2)}\right)}{2}-\bar{Y}\right\}^{2} \\
= & \frac{1}{4}\left\{E\left(\bar{y}_{S S}^{(1)}-\bar{Y}\right)^{2}+E\left(\bar{y}_{S S}^{(2)}-\bar{Y}\right)^{2}\right. \\
& \left.+2 E\left(\bar{y}_{S S}^{(1)}-\bar{Y}\right)\left(\bar{y}_{S S}^{(2)}-\bar{Y}\right)\right\}
\end{aligned}
$$

Since

$$
E\left(\bar{y}_{S S}^{(i)}-\bar{Y}\right)^{2}=\operatorname{MSE}\left(\bar{y}_{S S}^{(i)}\right)=\gamma_{m} \bar{Y}^{2}\left\{C_{y}^{2}+\nu C_{x}^{2}\left(A^{2} \nu-2 A K\right)\right\}, \quad i=1,2 .
$$

Let $\bar{y}_{1}=\bar{Y}+e_{0}^{(i)}$ and $\bar{x}_{i}=\bar{X}+e_{1}^{(i)}$ such that $E\left(e_{0}^{(i)}\right)=E\left(e_{1}^{(i)}\right)=0$, $i=1,2$. Then, $E\left(\bar{y}_{S S}^{(1)}-\bar{Y}\right)\left(\bar{y}_{S S}^{(2)}-\bar{Y}\right)$ up to the first order of approximation 
is

$$
\begin{aligned}
E\left(\bar{y}_{S S}^{(1)}-\bar{Y}\right)\left(\bar{y}_{S S}^{(2)}-\bar{Y}\right)= & E\left\{e_{0}^{(1)}-\frac{A \bar{Y} e_{1}^{(1)}}{(\bar{X}+\rho)}\right\}\left\{e_{0}^{(2)}-\frac{A \bar{Y} e_{1}^{(2)}}{(\bar{X}+\rho)}\right\} \\
= & E\left(e_{0}^{(1)} e_{0}^{(2)}\right)-\frac{A \bar{Y}\left\{E\left(e_{0}^{(2)} e_{1}^{(1)}\right)+E\left(e_{0}^{(1)} e_{1}^{(2)}\right)\right\}}{(\bar{X}+\rho)} \\
& +\frac{A^{2} \bar{Y}^{2} E\left(e_{1}^{(1)} e_{1}^{(2)}\right)}{(\bar{X}+\rho)^{2}}
\end{aligned}
$$

Substituting the results given in Sukhatme and Sukhatme (1997) $E\left(e_{0}^{(1)} e_{0}^{(2)}\right)=$ $-\bar{Y}^{2} C_{y}^{2} / N, E\left(e_{1}^{(1)} e_{1}^{(2)}\right)=-\bar{X}^{2} C_{x}^{2} / N, E\left(e_{0}^{(1)} e_{1}^{(2)}\right)=E\left(e_{0}^{(2)} e_{1}^{(1)}\right)=-\bar{X} \bar{Y} \rho C_{x} C_{y} / N$ we have

$$
\begin{aligned}
E\left(\bar{y}_{S S}^{(1)}-\bar{Y}\right)\left(\bar{y}_{S S}^{(2)}-\bar{Y}\right) & =-\frac{\bar{Y}^{2}\left\{C_{y}^{2}+A^{2} \nu^{2} C_{x}^{2}-2 A \nu \rho C_{x} C_{y}\right\}}{N} \\
& =-\frac{\bar{Y}^{2}\left\{C_{y}^{2}+\nu C_{x}^{2}\left(A^{2} \nu-2 A K\right)\right\}}{N}
\end{aligned}
$$

Putting the values from (17) and (18) in (16) we have

$$
\begin{aligned}
E\left(\widehat{\bar{Y}}_{S S}^{\prime}-\bar{Y}\right)^{2} & =\bar{Y}^{2} \frac{1}{4}\left[2\left(\frac{1}{m}-\frac{1}{N}\right)-\frac{2}{N}\right]\left\{C_{y}^{2}+\nu C_{x}^{2}\left(A^{2} \nu-2 A K\right)\right\} \\
& =\gamma_{2 m} \bar{Y}^{2}\left\{C_{y}^{2}+\nu C_{x}^{2}\left(A^{2} \nu-2 A K\right)\right\}
\end{aligned}
$$

Now consider

$$
\begin{aligned}
E\left(\bar{y}_{S S}^{(3)}-\bar{Y}\right)\left(\widehat{\bar{Y}}_{S S}^{\prime}-\bar{Y}\right)= & E\left\{\left(\bar{y}_{S S}^{(3)}-\bar{Y}\right)\left(\frac{\bar{y}_{S S}^{(1)}+\bar{y}_{S S}^{(2)}}{2}-\bar{Y}\right)\right\} \\
= & \frac{1}{2}\left\{E\left(\bar{y}_{S S}^{(3)}-\bar{Y}\right)\left(\bar{y}_{S S}^{(1)}-\bar{Y}\right)\right. \\
& \left.+E\left(\bar{y}_{S S}^{(3)}-\bar{Y}\right)\left(\bar{y}_{S S}^{(2)}-\bar{Y}\right)\right\}
\end{aligned}
$$


Since

$$
\begin{aligned}
E\left(\bar{y}_{S S}^{(3)}-\bar{Y}\right)\left(\bar{y}_{S S}^{(i)}-\bar{Y}\right)= & E\left\{e_{0}-\frac{A \bar{Y} e_{1}}{(\bar{X}+\rho)}\right\}\left\{e_{0}^{(i)}-\frac{A \bar{Y} e_{1}^{(i)}}{(\bar{X}+\rho)}\right\} \\
= & E\left(e_{0} e_{0}^{(i)}\right)-\frac{A \bar{Y}\left\{E\left(e_{0}^{(i)} e_{1}\right)+E\left(e_{0} e_{1}^{(i)}\right)\right\}}{(\bar{X}+\rho)} \\
& +\frac{A^{2} \bar{Y}^{2} E\left(e_{1} e_{1}^{(i)}\right)}{(\bar{X}+\rho)^{2}}
\end{aligned}
$$

where $i=1,2$.

Using the following results given in Sukhatme and Sukhatme (1997) $E\left(e_{0} e_{0}^{(i)}\right)=$ $\gamma_{2 m} \bar{Y}^{2} C_{y}^{2}, E\left(e_{1} e_{1}^{(i)}\right)=\gamma_{2 m} \bar{X}^{2} C_{x}^{2}, E\left(e_{0} e_{1}^{(i)}\right)=E\left(e_{0}^{(i)} e_{1}\right)=\gamma_{2 m} \bar{X} \bar{Y} \rho C_{x} C_{y}$ for $i=1,2$, we have

$$
E\left(\bar{y}_{S S}^{(3)}-\bar{Y}\right)\left(\bar{y}_{S S}^{(i)}-\bar{Y}\right)=\gamma_{2 m} \bar{Y}^{2}\left\{C_{y}^{2}+\nu C_{x}^{2}\left(A^{2} \nu-2 A K\right)\right\}
$$

Putting values from (15), (19), (20) in (14) we have

$$
\begin{aligned}
\operatorname{MSE}\left(\bar{y}_{S J}\right) & =(1-R)^{-2} \gamma_{2 m} \bar{Y}^{2}\left(1+R^{2}-2 R\right)\left\{C_{y}^{2}+\nu C_{x}^{2}\left(A^{2} \nu-2 A K\right)\right\} \\
& =\gamma_{n} \bar{Y}^{2}\left\{C_{y}^{2}+\nu C_{x}^{2}\left(A^{2} \nu-2 A K\right)\right\}
\end{aligned}
$$

which is equal to the mean square error of $\bar{y}_{S S}$. Also, the optimizing value of the characterizing scalar $A$ is given by $A=K / \nu=A_{o p t}$ and the minimum mean square error under optimizing value of $A=A_{o p t}$ is given by

$$
\operatorname{MSE}\left(\bar{y}_{S J}\right)_{\min }=\gamma_{n} \bar{Y}^{2}\left(1-\rho^{2}\right) C_{y}^{2}
$$

which is same as the mean square error of the linear regression estimator. Thus the proposed class of Jackknife sampling strategies is better than the strategy proposed by Singh and Tailor (2003) in the sense that the proposed class of estimators is almost unbiased and possesses an estimator having lesser mean square error.

\section{Midzuno - Lahiri - Sen Type Sampling Strat- egy}

Let us consider $\bar{y}_{S A}$ under Midzuno (1952) - Lahiri (1951) - Sen (1952) (MLS) type sampling scheme and denote it by $\bar{y}_{S M}$. The proposed MLS 
type sampling scheme for selecting a sample $S$ of size $n$ deals with selecting first unit with probability proportional to $\bar{X}+\rho+A\left(x_{i}-\bar{X}\right)$ where $x_{i}$ is the size of the first selected unit such that

$$
P(i)=\mathrm{P}\left(\text { selecting first unit } i \text { with size } x_{i}=\frac{\left\{\bar{X}+\rho+A\left(x_{i}-\bar{X}\right)\right\}}{\{N(\bar{X}+\rho)\}}\right.
$$

and selecting the remaining $n-1$ units in the sample from $N-1$ units in the population by simple random sampling without replacement. Thus the probability of selecting the sample size of $n$ can be expressed as

$$
P(s)=\frac{\{\bar{X}+\rho+A(\bar{x}-\bar{X})\}}{\left\{(\bar{X}+\rho)^{N} C_{n}\right\}}
$$

Consider

$$
\begin{aligned}
E\left(\bar{y}_{S M}\right) & =E\left[\frac{\bar{y}(\bar{X}+\rho)}{\{\bar{X}+\rho+A(\bar{x}-\bar{X})\}}\right] \\
& =\sum_{s=1}^{{ }^{N} C_{n}} \frac{\bar{y}(\bar{X}+\rho) P(s)}{\{\bar{X}+\rho+A(\bar{x}-\bar{X})\}} \\
& =\sum_{s=1}^{{ }^{N} C_{n}} \frac{\bar{y}}{{ }^{N} C_{n}}=E_{s}(\bar{y})=\bar{Y}
\end{aligned}
$$

showing that $\bar{y}_{S M}$ is an unbiased estimator of population mean $\bar{Y}$ for all values of $A$ under the proposed MLS type sampling scheme.

Now, in order to obtain MSE of the proposed class of estimators, let us consider

$$
\begin{aligned}
E\left(\bar{y}_{S M}^{2}\right) & =\sum_{s=1}^{{ }^{N} C_{n}} \bar{y}_{S M}^{2} \cdot P(s) \\
& =\sum_{s=1}^{{ }^{N} C_{n}} \frac{\bar{y}^{2}(\bar{X}+\rho)}{\{\bar{X}+\rho+A(\bar{x}-\bar{X})\}^{N} C_{n}} \\
& =E_{s}\left[\left(\bar{Y}+e_{0}\right)^{2}\left\{1+\frac{A e_{1}}{(\bar{X}+\rho)}\right\}^{-1}\right] \\
& =\bar{Y}^{2}+E_{s}\left(e_{0}^{2}\right)+\frac{A^{2} \bar{Y}^{2} E_{s}\left(e_{1}^{2}\right)}{(\bar{X}+\rho)^{2}}-\frac{2 A \bar{Y} E_{s}\left(e_{0} e_{1}\right)}{(\bar{X}+\rho)}
\end{aligned}
$$


(upto 1st order of approx). Therefore by using (6) we have

$$
\begin{aligned}
\operatorname{MSE}\left(\bar{y}_{S M}\right) & =\gamma_{n} \bar{Y}^{2}\left\{C_{y}^{2}+\nu C_{x}^{2}\left(A^{2} \nu-2 A K\right)\right\} \\
& =\operatorname{MSE}\left(\bar{y}_{S S}\right)=\operatorname{MSE}\left(\bar{y}_{S J}\right)=\operatorname{MSE}\left(\bar{y}_{S A}\right) \quad(\text { say })
\end{aligned}
$$

The optimizing value of the characterizing scalar $A$ is given by $A=K / \nu=$ $A_{\text {opt }}$ and the minimum mean square error under optimizing value of $A=A_{\text {opt }}$ is

$$
\begin{aligned}
\operatorname{MSE}\left(\bar{y}_{S M}\right)_{\min } & =\gamma_{n} \bar{Y}^{2}\left(1-\rho^{2}\right) C_{y}^{2} \\
& =\operatorname{MSE}\left(\bar{y}_{S S}\right)_{\min }=\operatorname{MSE}\left(\bar{y}_{S J}\right)_{\min }=\operatorname{MSE}\left(\bar{y}_{S A}\right)_{\min }
\end{aligned}
$$

which is same as the mean square error of the linear regression estimator. Thus the proposed class of MLS type sampling strategies is better than the strategy proposed by Singh and Tailor (2003) in the sense that the proposed class of estimators is unbiased and possesses an estimator having lesser mean square error.

\section{Concluding Remarks}

If the minimizing value $A=K / \nu=A_{\text {opt }}$ is known then, we have

$$
\begin{gathered}
\operatorname{MSE}(\bar{y})-M S E\left(\bar{y}_{S A}\right)_{\min }=\gamma_{n} \bar{Y}^{2} \rho^{2} C_{y}^{2} \geqslant 0 \\
\operatorname{MSE}\left(\bar{y}_{R}\right)-\operatorname{MSE}\left(\bar{y}_{S A}\right)_{\min }=\gamma_{n} \bar{Y}^{2}\left(C_{x}-\rho C_{y}\right)^{2} \geqslant 0 \\
\operatorname{MSE}\left(\bar{y}_{P}\right)-\operatorname{MSE}\left(\bar{y}_{S A}\right)_{\min }=\gamma_{n} \bar{Y}^{2}\left(C_{x}+\rho C_{y}\right)^{2} \geqslant 0 \\
\operatorname{MSE}\left(\bar{y}_{S R}\right)-\operatorname{MSE}\left(\bar{y}_{S A}\right)_{\min }=\gamma_{n} \bar{Y}^{2}\left(\nu C_{x}-\rho C_{y}\right)^{2} \geqslant 0 \\
\operatorname{MSE}\left(\bar{y}_{S P}\right)-\operatorname{MSE}\left(\bar{y}_{S}\right)_{\min }=\gamma_{n} \bar{Y}^{2}\left(\nu C_{x}+\rho C_{y}\right)^{2} \geqslant 0
\end{gathered}
$$

where $\bar{y}_{R}=\bar{y} \bar{X} / \bar{x}, \bar{y}_{P}=\bar{y} \bar{x} / \bar{X}$ and $\bar{y}_{l r}=\bar{y}+b(\bar{X}-\bar{x})$ are classical ratio, product and linear regression estimators respectively.

Hence, under the optimizing value of the characterizing scalar $A=K / \nu=$ $A_{\text {opt }}$, the mean square error of the proposed sampling strategies is always lesser than that of $\bar{y}, \bar{y}_{S R}, \bar{y}_{S P}, \bar{y}_{P}$ and $\bar{y}_{R}$. Therefore, the proposed sampling strategies are always better than $\bar{y}, \bar{y}_{S R}, \bar{y}_{S P}, \bar{y}_{P}, \bar{y}_{R}$ and $\bar{y}_{l r}$ both in sense of unbiasedness and gain in efficiency. 
Table 1. Bias, Mean Square Error and Percent Relative Efficiency

\begin{tabular}{|c|c|c|c|c|c|c|c|}
\hline Estimator $(t)$ & $\bar{y}$ & $\bar{y}_{R}$ & $\bar{y}_{P}$ & $\bar{y}_{l r}$ & $\bar{y}_{S P}$ & $\bar{y}_{S R}$ & $\begin{array}{l}\bar{y}_{S M} \\
\bar{y}_{S J} \\
\end{array}$ \\
\hline Bias (t) & 0 & -244.68 & 3376.77 & 3867.58 & 3247.27 & -350.81 & 0 \\
\hline MSE (t) & 6564590 & 1249925 & 21072230 & 1221872 & 20346316 & 1284228 & 1221872 \\
\hline $\operatorname{PRE}(\mathrm{t}: \bar{y})$ & 100 & 525.20 & 31.15 & 537.26 & 32.26 & 511.1697 & 537.26 \\
\hline
\end{tabular}

\section{Empirical Study}

Here an empirical study has been carried out on an example considered by Singh and Chaudhary (1986) is given, wherein the following values are obtained $\bar{Y}=1467.545, \bar{X}=22.62, C_{x}=1.460904, C_{y}=1.7459, \rho=0.9022$. The bias, mean square error and percent relative efficiency (PRE) of the sample mean $\bar{y}$, ratio estimator $\bar{y}_{R}$, product estimator $\bar{y}_{P}, \bar{y}_{S P}, \bar{y}_{S R}$ and $\bar{y}_{l r}$ are given in Table 1.

It should be noted that the above results are scaled by the factor $\gamma_{n}$. Further, it can be easily observed from the table that only sample mean $\bar{y}$ and the proposed sampling strategies are unbiased. Further, it can be seen that $\bar{y}_{l r}$ and the proposed sampling strategies attains the minimum mean square error but $\bar{y}_{l r}$ is biased. It is evident from the above empirical study that under the optimizing value of the proposed sampling strategies are better than the remaining sampling strategies both in terms of unbiasedness and mean square error.

\section{Acknowledgment}

The valuable suggestions by the referee are gratefully acknowledged.

\section{References}

Cochran, W.G. (1977). Sampling Techniques. John Wiley and Sons, New York.

Gray, H.L. and Schucany, W.R. (1972). The Generalized Jack-knife Statistic. Marcel Dekker, New York.

Lahiri, D.B. (1951). A method of sample selection providing unbiased ratio estimates. Bull. Int. Stat. Inst., 3, 133-140. 
Midzuno, H. (1952). On the sampling system with probability proportional to the sum of the sizes. Ann. Inst. Stat. Math., 3, 99-107.

Quenouille, M.H. (1956). Notes on bias in estimation. Biometrika, 43, 353-360.

Sen, A.R. (1952). Present status of probability sampling and its use in the estimation of a characteristic. Econometrika, 20,103.

Singh, D. and Chaudhary, F.S. (2002). Theory and Analysis of Sampling Survey Design. New Age Pvt. Limited, New Delhi.

Singh, H.P. and Tailor, R. (2003). Use of known correlation coefficient in estimating the finite population mean. Statistics in Transition, 6, 555-560.

Sukhatme, P.V. and Sukhatme, B.V. (1997). Sampling Theory of Surveys with Applications. Piyush Publications, Delhi.

Walsh, J.E. (1970). Generalization of ratio estimator for population total. Sankhya, A, 32, 99-106.

\section{Shashi Bhushan}

Department of Statistics,

PUC-Campus,

Mizoram University,

Aizawl-796001,

Mizoram, India.

email: shashi.py@gmail.com

email:dr.shashibhushan@ymail.com

\section{Arvind Pandey}

Department of Statistics,

PUC-Campus,

Mizoram University,

Aizawl-796001,

Mizoram, India. 